\title{
Quantizers with Uniform Encoders and Channel Optimized Decoders *
}

\author{
Benjamin Farber and Kenneth Zeger \\ University of California, San Diego
}

\begin{abstract}
Scalar quantizers with uniform encoders and channel optimized decoders are studied for uniform sources and binary symmetric channels. It is shown that the Natural Binary Code and Folded Binary Code induce point density functions that are uniform on proper subintervals of the source support, whereas the Gray Code does not induce a point density function. The mean squared errors for the Natural Binary Code, Folded Binary Code, Gray Code, and for randomly chosen index assignments are calculated and the Natural Binary Code is shown to be mean squared optimal among all possible index assignments. In contrast, it is shown that almost all index assignments perform poorly and have degenerate codebooks.
\end{abstract}

\section{Introduction}

The most basic source and quantizer are the uniform scalar source and the uniform scalar quantizer. If the source is uniform on $[0,1]$, for example, then an $n$-bit uniform quantizer has equally spaced encoding cells of size $2^{-n}$ and has equally spaced output points which are the centers of the encoding cells. For this source, the mean squared distortion of this quantizer is known exactly when there is no channel noise, and is known to be minimal among all quantizers.

In the presence of channel noise, one approach to improving system performance is to add explicit error control coding, so that some of the transmission rate is devoted towards source coding and some towards channel coding. Drawbacks of this include the added complexity and delay of channel decoding.

An alternative low-complexity approach in the presence of channel noise is to add to the quantizer an index assignment, which permutes the binary words associated with each encoding cell prior to transmission over the channel, and then unpermutes the binary words at the receiver prior to assigning a reproduction point at the output. The cells are assumed to be labeled in increasing order from left to right, before the index assignment. Examples

${ }^{*}$ Supported in part by the National Science Foundation. The authors are with the Department of Electrical and Computer Engineering, University of California, San Diego, 9500 Gilman Dr. MC 0407, La Jolla, CA 92093-0407. Telephone: 858-822-0440 (Zeger), 858-822-1250 (Farber). Email: \{farber, zeger\}@code.ucsd.edu 
of index assignments include the Natural Binary Code, the Folded Binary Code, and the Gray Code. The benefit of an index assignment is derived from the fact that reproduction codepoints that are relatively close on the real line can be assigned binary words which are close in the Hamming sense (i.e. in the number of same bits) on average. Thus when channel errors occur, the mean squared error impact on the quantizer is reduced.

Yamaguchi and Huang [6] and Huang [7] derived formulas for the mean squared error of uniform scalar quantizers and uniform sources for the Natural Binary Code, the Gray Code, and for a randomly chosen index assignment on a binary symmetric channel. They also asserted (without a published proof) the optimality of the Natural Binary Code for the binary symmetric channel. Crimmins et al. [1] studied the uniform scalar quantizer for the uniform source and proved the Yamaguchi-Huang assertion, that the Natural Binary Code is the best possible index assignment in the mean squared sense for the binary symmetric channel. McLaughlin, Neuhoff, and Ashley [2] generalized this result for certain uniform vector quantizers and uniform vector sources. Other than these papers, there are no others presently known in the literature giving index assignment optimality results. In [3] explicit mean squared error formulas were computed for uniform sources on binary asymmetric channels with various structured classes of index assignments. In [4] it was shown that for the uniform source and uniform quantizer the mean squared error resulting from a randomly chosen index assignment was, on average, equal in the limit of large $n$ to that of the worst possible index assignment. In this sense the result showed that randomly chosen index assignments are asymptotically bad. A number of papers have also studied algorithmic techniques for designing good index assignments for particular sources and channels (see the citations in [5, p. 2372]).

While index assignments can improve the robustness of quantizers designed for noiseless channels to the degradation caused by channel noise, another low-complexity approach is to use quantizers whose encoders and/or decoders are designed for the channel's statistical behavior. It is known that an optimal quantizer for a noiseless channel must satisfy what are known as "nearest neighbor" and "centroid" conditions on its encoder and decoder, respectively. For discrete memoryless channels it is known that an optimal quantizer must satisfy what we call "weighted nearest neighbor" and "weighted centroid" conditions on its encoder and decoder, respectively (see [8] for example). Even for uniform scalar sources, the resulting quantizers in general do not have uniform encoding cells nor equally spaced reproduction codepoints. In fact very little is presently understood analytically about quantizers for noisy channels beyond the Natural Binary Code optimality results previously mentioned for uniform quantizers.

In the present paper, we attempt to move a step closer towards understanding optimal quantization for noisy channels by examining the structure of quantizers with uniform encoders and channel optimized decoders (i.e. that satisfy the weighted centroid condition), for uniform sources on $[0,1]$ and for certain previously studied index assignments. In particular, we study the high resolution distribution of codepoints for such quantizers and the resulting distortions. Slightly more general, but notationally cumbersome results could also be easily obtained from our results by allowing the source to be confined to any bounded interval instead of just $[0,1]$.

An important tool in analyzing the performance of quantizers is the concept of point density functions. Point density functions characterize the high resolution distribution of scalar quantizer codepoints. As a result, they provide insight about the asymptotic behavior 
of scalar quantizer codebooks and encoding cells. Point density functions also are useful in analyzing the distortion of quantizers. For example, Bennett's integral gives the average distortion in the high resolution case for a nonuniform quantizer in terms of a point density function, source distribution, and size of the quantizer codebook (see [5] for more details). For uniform quantizers, the computation of a point density function is trivial. For nonuniform quantizers however, point density functions are not always guaranteed to exist, and when they do, their computation can be difficult.

Point density functions depend on the quantizer decoders. Channel optimized quantizer decoders, in turn, depend on the source, the quantizer encoder, the channel, and the index assignment. For this paper, we assume a uniform source on [0,1], a uniform quantizer encoder, a channel optimized quantizer decoder, and a binary symmetric channel with bit error probability $\epsilon$. An index assignment maps source codewords to channel codewords. The quantizer has $2^{n}$ encoding cells, and index assignments are one-to-one maps from the index of an encoding cell to a binary word of length $n$. These words are transmitted across the channel and decoded according to the weighted centroid condition.

Certain results we obtain are somewhat counter-intuitive. For example, we show that for a binary symmetric channel with bit error probability $\epsilon$, quantizers using the Natural Binary Code index assignment and $n$ bits of resolution have codepoints uniformly distributed on the interval $[\epsilon+\delta, 1-\epsilon-\delta]$ where $\delta=(1-2 \epsilon) / 2^{n+1}$. This is peculiar in light of the fact that the source is uniformly distributed on the interval $[0,1]$, and yet asymptotically as $n \rightarrow \infty$ no codepoints are located within a distance of $\epsilon$ from 0 or 1 . The lack of codepoints in regions of positive source probability is due to the reduction in average distortion that results from moving codepoints closer to the source mean (by the weighted centroid condition), to avoid large jumps in Euclidean distance from channel errors. The weighted centroid condition dictates this movement of codepoints to minimize average distortion for a given quantizer encoder. A similar result occurs for the Folded Binary Code. For the Gray Code index assignment, we show that in fact no point density function exists.

We also show that asymptotically, almost all index assignments give rise to quantizers which have almost all of their codepoints clustered very close to the source's mean value (i.e. 1/2). Thus almost all index assignments are bad. As $n$ grows, the clustering of codepoints becomes tighter and tighter. This contrasts with the Natural Binary Code and the Folded Binary Code cases where the codepoints remain uniformly distributed on proper subsets of $[0,1]$ no matter how large $n$ becomes. An additional curiosity we show is that among all possible index assignments, the Natural Binary Code is optimal despite its lack of codepoints within $\epsilon$ of 0 or 1 .

Our main results for quantizers with uniform encoders and channel optimized decoders are the following. First, we show that the Natural Binary Code index assignment yields a uniform point density function on the interval $(\epsilon, 1-\epsilon)$ (Theorem 3.1), the Folded Binary Code index assignment yields a uniform point density function on a union of two proper subintervals of $[0,1]$ (Theorem 4.1), the Gray Code index assignment does not yield a point density function (Theorem 5.1), and an arbitrarily large fraction of all index assignments have an arbitrarily large fraction of codepoints arbitrarily close to the source mean as $n \rightarrow \infty$ (Theorem 6.1). Then we extend a result in [4] by showing that most index assignments are asymptotically bad (Theorem 7.1), and we extend results in [3], [6], and [7] by computing the mean squared error resulting from the Natural Binary Code (Theorem 7.3), the Folded Binary Code (Theorem 7.5), the Gray Code (Theorem 7.7), and a 
randomly chosen index assignment (Theorem 7.9). As comparisons, we state previously known mean squared error formulas for channel unoptimized decoders (i.e. that satisfy the centroid condition), for the Natural Binary Code (Theorem 7.2), the Folded Binary Code (Theorem 7.4), the Gray Code (Theorem 7.6), and for a randomly chosen index assignment (Theorem 7.8). Finally we extend the (uniform scalar quantizer) proof in [2] by showing that the Natural Binary Code is an optimal index assignment (Theorem 7.10).

In order to conserve space, we have provided proof sketches to only two results.

\section{Preliminaries}

A rate $n$ quantizer on $[0,1]$ is a mapping $\mathcal{Q}:[0,1] \longrightarrow\left\{y_{n}(0), y_{n}(1), \ldots, y_{n}\left(2^{n}-1\right)\right\}$. The real-valued quantities $y_{n}(i)$ are called codepoints and the set $\left\{y_{n}(0), \ldots, y_{n}\left(2^{n}-1\right)\right\}$ is called a codebook. For a noiseless channel, the quantizer $\mathcal{Q}$ is the composition of a quantizer encoder and a quantizer decoder. These are respectively mappings $\mathcal{Q}_{e}$ : $[0,1] \longrightarrow\left\{0,1, \ldots, 2^{n}-1\right\}$ and $\mathcal{Q}_{d}:\left\{0,1, \ldots, 2^{n}-1\right\} \longrightarrow\left\{y_{n}(0), y_{n}(1), \ldots, y_{n}\left(2^{n}-1\right)\right\}$ such that $\mathcal{Q}_{d}(i)=y_{n}(i)$ for all $i$. For each $i$ the set $\mathcal{Q}^{-1}\left(y_{n}(i)\right)=\mathcal{Q}_{e}^{-1}\left(\mathcal{Q}_{d}^{-1}\left(y_{n}(i)\right)\right)$ is called the $i$ th encoding cell. The quantizer encoder is said to be uniform if for each $i$, $\mathcal{Q}^{-1}\left(y_{n}(i)\right) \supseteq\left(i 2^{-n},(i+1) 2^{-n}\right)$.

The nearest neighbor cells of a rate $n$ quantizer are the sets $R_{n}(i)=\left\{x:\left|y_{n}(i)-x\right|<\right.$ $\left.\left|y_{n}(j)-x\right|, \forall j \neq i\right\}$ for $0 \leq i \leq 2^{n}-1$. Let $m$ denote Lebesgue measure and for each $i$ let $\mu_{n}(i)=m\left(R_{n}(i)\right)$. A quantizer's encoder is said to satisfy the nearest neighbor condition if for each $i, \mathcal{Q}^{-1}\left(y_{n}(i)\right) \supseteq R_{n}(i)$. That is, its encoding cells are essentially nearest neighbor cells (boundary points can be assigned arbitrarily).

For a given $n, i$, and source random variable $X$, the centroid of the $i$ th cell of the quantizer $\mathcal{Q}$ is the conditional mean $c_{n}(i)=E\left[X \mid \mathcal{Q}(X)=y_{n}(i)\right]$. The quantizer decoder is said to satisfy the centroid condition if the codepoints satisfy $y_{n}(i)=c_{n}(i)$ for all $i$. A quantizer is uniform if the encoder is uniform and for each $i$ the decoder codepoint $y_{n}(i)$ is the midpoint of the cell $\mathcal{Q}^{-1}\left(y_{n}(i)\right)$. In particular, if the source is uniform, then a uniform quantizer satisfies the nearest neighbor and centroid conditions.

For a rate $n$ quantizer, an index assignment $\pi_{n}$ is a permutation of the set $\left\{0,1, \ldots, 2^{n}-\right.$ $1\}$. Let $S_{2^{n}}$ denote the set of all $2^{n}$ ! such permutations. For a noisy channel, a random variable $X \in[0,1]$ is quantized by transmitting the index $I=\pi_{n}\left(\mathcal{Q}_{e}(X)\right)$ across the channel, receiving index $J$ from the channel, and then decoding the codepoint $y_{n}\left(\pi_{n}^{-1}(J)\right)=$ $\mathcal{Q}_{d}\left(\pi_{n}^{-1}(J)\right)$. We impose the following monotonicity constraint on quantizer encoders in order to be able to unambiguously refer to certain index assignments: For all $s, t \in[0,1]$, if $s<t$, then $\mathcal{Q}_{e}(s) \leq \mathcal{Q}_{e}(t)$. The mean squared error (MSE) is defined as $D=$ $E\left[\left(X-\mathcal{Q}_{d}\left(\pi_{n}^{-1}(J)\right)\right)^{2}\right]$. The random index $J$ is a function of the source random variable $X$, the randomness in the channel, and the deterministic functions $\mathcal{Q}_{e}$ and $\pi_{n}$.

Assume a binary symmetric channel with bit error probability $\epsilon$. Denote the probability that index $j$ was received, given that index $i$ was sent by $p(j \mid i)=\epsilon^{H_{n}(i, j)}(1-\epsilon)^{n-H_{n}(i, j)}$ for $0 \leq \epsilon \leq 1 / 2$, where $H_{n}(i, j)$ is the Hamming distance between $n$-bit binary words $i$ and $j$. Let $q(i \mid j)$ denote the probability that index $i$ was sent, given that index $j$ was received.

For a given source $X$, channel $p(\cdot \mid \cdot)$, index assignment $\pi_{n}$, and quantizer encoder, the 
quantizer decoder is said to satisfy the weighted centroid condition if the codepoints satisfy

$$
y_{n}(j)=\sum_{i=0}^{2^{n}-1} c_{n}(i) q\left(\pi_{n}(i) \mid \pi_{n}(j)\right) .
$$

Throughout this paper we assume a uniform quantizer encoder, so the centroids of the encoder cells are given by $c_{n}(i)=(i+(1 / 2)) 2^{-n}$ for $0 \leq i \leq 2^{n}-1$. For a given quantizer encoder and index assignment, we say the quantizer decoder is channel optimized if it satisfies the weighted centroid condition. Since a quantizer decoder obeying the weighted centroid condition minimizes the mean squared error for a given quantizer encoder and index assignment, we are also using channel optimized to denote a MMSE quantizer decoder.

For any set $A$, let $\mathcal{I}_{A}(x)$ be the indicator function of $A$. For each $n$ and each index assignment $\pi_{n} \in S_{2^{n}}$, define the function $\lambda_{\pi_{n}}^{(n)}:[0,1] \rightarrow[0, \infty)$ by

$$
\lambda_{\pi_{n}}^{(n)}(x)=\sum_{i=0}^{2^{n}-1} \frac{1}{2^{n} \mu_{n}(i)} \mathcal{I}_{R_{n}(i)}(x) .
$$

For a sequence $\pi_{n} \in S_{2^{n}}$ (for $n=1,2, \ldots$ ) of index assignments, if there exists a function $\lambda$ such that $\lambda(x)=\lim _{n \rightarrow \infty} \lambda_{\pi_{n}}^{(n)}(x) \quad \forall x \in[0,1]$ and $\int_{0}^{1} \lambda(x) d x=1$ then $\lambda$ is said to be a point density function with respect to $\left\{\pi_{n}\right\}$.

Let a channel optimized uniform quantizer denote a rate $n$ quantizer with a uniform encoder on $[0,1]$ and a channel optimized decoder, along with a uniform source on $[0,1]$, and a binary symmetric channel with bit error probability $\epsilon$. Let a channel unoptimized uniform quantizer denote a rate $n$ uniform quantizer on $[0,1]$, along with a uniform source on $[0,1]$, and a binary symmetric channel with bit error probability $\epsilon$.

\section{Natural Binary Code Index Assignment}

For each $n$, the Natural Binary Code (NBC) is the index assignment defined by

$$
\pi_{n}^{(N B C)}(i)=i \text { for } 0 \leq i \leq 2^{n}-1
$$

The following theorem shows that with the Natural Binary Code, the quantizer codepoints are uniformly distributed on a proper subinterval in the source's support region, in the limit of high resolution. As the channel improves (i.e. as $\epsilon \rightarrow 0$ ), the point density function approaches a uniform distribution on $[0,1]$.

Theorem 3.1 A sequence of channel optimized uniform quantizers with the Natural Binary Code index assignment has a point density function given by

$$
\lambda(x)=\left\{\begin{array}{cl}
\frac{1}{1-2 \epsilon} & \text { if } \epsilon<x<1-\epsilon \\
0 & \text { otherwise }
\end{array}\right.
$$




\section{Folded Binary Code Index Assignment}

For each $n$, the Folded Binary Code (FBC) is the index assignment defined by

$$
\pi_{n}^{(F B C)}(i)=\left\{\begin{array}{cll}
2^{n-1}-1-i & \text { if } & 0 \leq i \leq 2^{n-1}-1 \\
i & \text { if } & 2^{n-1} \leq i \leq 2^{n}-1
\end{array}\right.
$$

The following theorem shows that with the Folded Binary Code, the quantizer codepoints are uniformly distributed on two proper subintervals of the source's support region, in the limit of high resolution.

Theorem 4.1 A sequence of channel optimized uniform quantizers with the Folded Binary Code index assignment has a point density function given by

$$
\lambda(x)=\left\{\begin{array}{cl}
\frac{1}{(1-2 \epsilon)^{2}} & \text { if } \frac{3 \epsilon-2 \epsilon^{2}}{2}<x<\frac{1-\epsilon+2 \epsilon^{2}}{2} \text { or } \frac{1+\epsilon-2 \epsilon^{2}}{2}<x<1-\left(\frac{3 \epsilon-2 \epsilon^{2}}{2}\right) . \\
0 & \text { otherwise }
\end{array}\right.
$$

\section{Gray Code Index Assignment}

For each $n$, let $\pi_{n}^{(G C)}$ denote the Gray Code (GC) index assignment, recursively defined by

$$
\begin{aligned}
& \pi_{1}^{(G C)}(0)=0 \\
& \pi_{1}^{(G C)}(1)=1 \\
& \pi_{n+1}^{(G C)}(i)= \begin{cases}\pi_{n}^{(G C)}(i) & \text { if } 0 \leq i \leq 2^{n}-1 \\
\pi_{n}^{(G C)}\left(2^{n+1}-1-i\right)+2^{n} & \text { if } 2^{n} \leq i \leq 2^{n+1}-1 .\end{cases}
\end{aligned}
$$

Theorem 5.1 A sequence of channel optimized uniform quantizers with the Gray Code index assignment does not have a point density function.

\section{Randomly Chosen Index Assignments}

Suppose for each $n \geq 1$ an index assignment $\Pi_{n}$ is chosen uniformly at random from the set of all $2^{n}$ ! index assignments. Then $\lambda$ does not exist in a deterministic sense as the limit of $\lambda_{\Pi_{n}}^{(n)}$. However, the distribution of codepoints can still be characterized probabilistically.

The theorem below shows that asymptotically, an arbitrarily large fraction of index assignments induce an arbitrarily large fraction of codepoints to be arbitrarily close to $1 / 2$. This result is in contrast to the fact that the Natural Binary Code index assignment has an arbitrarily small fraction of codepoints arbitrarily close to $1 / 2$.

Theorem 6.1 For a channel optimized uniform quantizer, arbitrarily small $r, s, t>0$, and $n$ sufficiently large, at least $(1-r) 2^{n}$ ! index assignments each have at least $(1-s) 2^{n}$ codepoints within a distance of $t$ from $1 / 2$. 
Proof: Assume $\Pi_{n}$ is chosen uniformly at random from the set $S_{2^{n}}$ of all $2^{n}$ ! index assignments. Let $\delta=\epsilon /(1-\epsilon)$ and note that $(1-\epsilon)(1+\delta)=1$. Also, let

$$
a_{n}=\left(c_{n}(j)-\frac{1}{2}\right)\left(\frac{1}{1-2^{-n}}\right)\left((1-\epsilon)^{n}-2^{-n}\right) .
$$

By the Chebychev inequality, for any $t>0$, it can be shown that

$$
\frac{1}{2^{n !}}\left|\left\{\pi_{n} \in S_{2^{n}}:\left|y_{n}(j)-\frac{1}{2}\right|>t\right\}\right|<\frac{\operatorname{Var}\left(y_{n}(j)\right)}{\left(t-\left|a_{n}\right|\right)^{2}} .
$$

Thus, for any $A>0$ there are at most $\frac{2^{n} ! 2^{n}}{A} \cdot \frac{\operatorname{Var}\left(y_{n}(j)\right)}{\left(t-\left|a_{n}\right|\right)^{2}}$ index assignments $\pi_{n} \in S_{2^{n}}$, such that for each such $\pi_{n}$, there exist at least $A$ codepoints $y_{n}(j)$ satisfying $\left|y_{n}(j)-\frac{1}{2}\right|>t$. Taking $A=\alpha 2^{n}$ we get the following equivalent conclusion. For any $\alpha \in(0,1)$, there are at least $2^{n} !\left(1-\frac{\operatorname{Var}\left(y_{n}(j)\right)}{\alpha\left(t-\left|a_{n}\right|\right)^{2}}\right)$ index assignments $\pi_{n} \in S_{2^{n}}$ such that for each such $\pi_{n}$, there exist at most $\alpha 2^{n}$ codepoints $y_{n}(j)$ satisfying $\left|y_{n}(j)-\frac{1}{2}\right|>t$.

A careful look at the variance shows a dependency on $j$ but we can easily make a uniform upper bound on the variance which goes to zero at the speed $O\left(2^{-\beta n}\right)$, where $\beta=-\log _{2}\left(1-2 \epsilon+2 \epsilon^{2}\right)>0$. We choose $t=\alpha=2^{-\beta n / 4}$. This implies that for any $n$, a fraction of at least $1-O\left(2^{-\beta n / 4}\right)$ of all index assignments have the property that the fraction of codepoints $y_{n}(j)$ farther from $1 / 2$ than $2^{-\beta n / 4}$, is at most $2^{-\beta n / 4}$.

\section{Distortion Analysis}

Let $\pi_{n}$ be the index assignment for a rate $n$ quantizer with a uniform encoder on $[0,1]$ for a uniform source on $[0,1]$ and a binary symmetric channel with bit error probability $\epsilon$. Then the end-to-end MSE can be written as

$$
D^{\left(\pi_{n}\right)}=\sum_{i=0}^{2^{n}-1} \sum_{j=0}^{2^{n}-1} p\left(\pi_{n}(j) \mid \pi_{n}(i)\right) \int_{i / 2^{n}}^{(i+1) / 2^{n}}\left(x-y_{n}(j)\right)^{2} d x .
$$

For any index assignment $\pi_{n} \in S_{2^{n}}$, let $D_{C U}^{\left(\pi_{n}\right)}$ denote the MSE of a channel unoptimized uniform quantizer and let $D_{C O}^{\left(\pi_{n}\right)}$ denote the MSE of a channel optimized uniform quantizer. A sequence of index assignments $\pi_{n} \in S_{2^{n}}$ is said to be optimal for a channel unoptimized uniform quantizer if for all $n$ and for all $\pi_{n}^{\prime} \in S_{2^{n}}, D_{C U}^{\left(\pi_{n}\right)} \leq D_{C U}^{\left(\pi_{n}^{\prime}\right)}$ and is said to be optimal for a channel optimized uniform quantizer if for all $n$ and for all $\pi_{n}^{\prime} \in S_{2^{n}}, D_{C O}^{\left(\pi_{n}\right)} \leq D_{C O}^{\left(\pi_{n}^{\prime}\right)}$.

In [4] it was shown that randomly chosen index assignments for a channel unoptimized uniform quantizer are asymptotically bad in the sense that their MSE approaches that of the worst possible index assignment in the limit as $n \rightarrow \infty$. The following theorem extends the result to a channel optimized uniform quantizer.

Theorem 7.1 The mean squared error of a channel optimized uniform quantizer is at most $1 / 12$, and for $n$ sufficiently large, an arbitrarily large fraction of index assignments achieve a mean squared error arbitrarily close to $1 / 12$. 
Although Theorem 7.1 indicates that asymptotically most index assignments yield mean squared errors close to $1 / 12$, in the following it will be shown that the Natural Binary Code, the Folded Binary Code, and the Gray Code perform substantially better asymptotically.

The next two theorems give the mean squared errors for the Natural Binary Code with a channel unoptimized decoder and with a channel optimized decoder. Theorem 7.2 was stated in [6] (see, e.g. [3] for a proof). The results are given as a function of the quantizer rate $n$ and the channel bit error probability $\epsilon$. Analogous results are then given for the Folded Binary Code, the Gray Code, and the average for an index assignment chosen uniformly at random.

Theorem 7.2 The mean squared error of a channel unoptimized uniform quantizer with the Natural Binary Code index assignment is

$$
D_{C U}^{(N B C)}=\frac{2^{-2 n}}{12}+\frac{\epsilon}{3}\left(1-2^{-2 n}\right)
$$

Theorem 7.3 The mean squared error of a channel optimized uniform quantizer with the Natural Binary Code index assignment is

$$
D_{C O}^{(N B C)}=\frac{2^{-2 n}}{12}+\frac{\epsilon(1-\epsilon)}{3}\left(1-2^{-2 n}\right)
$$

The next two theorems give the mean squared errors for the Folded Binary Code with a channel unoptimized decoder and with a channel optimized decoder. Theorem 7.4 was given in [3].

Theorem 7.4 The mean squared error of a channel unoptimized uniform quantizer with the Folded Binary Code index assignment is

$$
D_{C U}^{(F B C)}=\frac{1}{12}\left(5 \epsilon-2 \epsilon^{2}+2^{-2 n}\left(1-8 \epsilon+8 \epsilon^{2}\right)\right)
$$

Theorem 7.5 The mean squared error of a channel optimized uniform quantizer with the Folded Binary Code index assignment is

$$
D_{C O}^{(F B C)}=\frac{1}{12}\left(5 \epsilon-9 \epsilon^{2}+8 \epsilon^{3}-4 \epsilon^{4}-2^{-2 n}(1-2 \epsilon)^{4}\right) .
$$

The next two theorems give the mean squared errors for the Gray Code with a channel unoptimized decoder and with a channel optimized decoder. Theorem 7.6 was stated in [7] (see, e.g. [3] for a proof).

Theorem 7.6 The mean squared error of a channel unoptimized uniform quantizer with the Gray Code index assignment is

$$
D_{C U}^{(G C)}=\frac{1}{6}-\frac{2^{-2 n}}{12}-\frac{\left(\frac{1}{4}-\frac{\epsilon}{2}\right)\left(1-\left(\frac{1}{4}-\frac{\epsilon}{2}\right)^{n}\right)}{\frac{3}{2}+\epsilon} .
$$


Theorem 7.7 The mean squared error of a channel optimized uniform quantizer with the Gray Code index assignment is

$$
D_{C O}^{(G C)}=\frac{1}{12}-\frac{1}{4} \cdot \frac{1-\left(\frac{1}{2}-\epsilon\right)^{2 n}}{\left(\frac{1}{2}-\epsilon\right)^{-2}-1} .
$$

It can be seen from Theorem 7.2 and Theorem 7.3 that for the NBC, the reduction in MSE obtained by using a channel optimized quantizer decoder instead of one obeying the centroid condition, is $\epsilon^{2}\left(1-2^{-2 n}\right) / 3$. For small $\epsilon$, the MSE reduction is thus small. For a randomly chosen index assignment however, Theorem 7.8 and Theorem 7.9 show that channel optimized decoders reduce the average distortion by a factor of two over decoders obeying the centroid condition, independent of $\epsilon$, in the limit as $n \rightarrow \infty$. Theorem 7.8 was stated in [6], and [4] contains a concise proof. Let $D_{C U}^{(R A N)}$ be a random variable denoting the MSE of a channel unoptimized uniform quantizer with a randomly chosen index assignment.

Theorem 7.8 The average mean squared error of a channel unoptimized uniform quantizer with an index assignment chosen uniformly at random is

$$
E\left[D_{C U}^{(R A N)}\right]=\frac{2^{-2 n}}{12}+\frac{1}{6}+\frac{1-\left(2^{n}+1\right)(1-\epsilon)^{n}}{6 \cdot 2^{n}} .
$$

Since most index assignments are asymptotically bad, their average is bad as well. More precisely, the next theorem shows that the average asymptotic MSE of a channel optimized uniform quantizer with an arbitrary index assignment converges to $1 / 12$, consistent with Theorem 7.1. Let $D_{C O}^{(R A N)}$ be a random variable denoting the MSE of a channel optimized uniform quantizer with a randomly chosen index assignment.

Theorem 7.9 The average mean squared error of a channel optimized uniform quantizer with an index assignment chosen uniformly at random is

$$
E\left[D_{C O}^{(R A N)}\right]=\frac{2^{-2 n}}{12}+\frac{1}{12}+\frac{1-\left(2^{n}+1\right)\left(1-2 \epsilon+2 \epsilon^{2}\right)^{n}}{12 \cdot 2^{n}}
$$

Crimmins et al. [1] and McLaughlin, Neuhoff, and Ashley [2] showed that the Natural Binary Code is optimal for a channel unoptimized uniform quantizer. We next extend the proof in [2] to show that the Natural Binary Code is also optimal for a channel optimized uniform quantizer.

Theorem 7.10 The Natural Binary Code index assignment is optimal for a channel optimized uniform quantizer.

Proof: Let $\underline{c}=\left[c_{n}(0), c_{n}(1), \ldots, c_{n}\left(2^{n}-1\right)\right]^{t}$ and $\underline{y}=\left[y_{n}(0), y_{n}(1), \ldots, y_{n}\left(2^{n}-1\right)\right]^{t}$ denote the column vectors of cell centroids and codepoints, respectively, and let $Q_{\pi_{n}}$ denote the $2^{n} \times 2^{n}$ matrix whose $(i, j)^{t h}$ elements are $q\left(\pi_{n}(i) \mid \pi_{n}(j)\right)$. Then it can be shown that

$$
D_{C O}^{\left(\pi_{n}\right)}=\frac{1}{3}-2^{-n}\|\underline{y}\|^{2}
$$




$$
\begin{aligned}
& =\frac{1}{3}-2^{-n} \underline{c}^{t} P Q_{\pi_{n}^{(N B C)}}^{2} P^{t} \underline{c} \\
& =\frac{1}{3}-2^{-n} \underline{z}^{t} Q_{\pi_{n}^{(N B C)} \underline{z}}^{2} \\
& =\frac{1}{3}-2^{-n} \underline{z}^{t} \hat{Q}_{\pi_{n}^{(N B C)} \underline{z}}
\end{aligned}
$$

where $P$ is a $2^{n} \times 2^{n}$ permutation matrix such that $Q_{\pi_{n}}^{2}=P Q_{\pi_{n}^{(N B C)}}^{2} P^{t}$, where $\underline{z}=P^{t} \underline{c}$, and where $\hat{Q}_{\pi_{n}^{(N B C)}}$ is the same as $Q_{\pi_{n}^{(N B C)}}$ but with $\epsilon$ replaced by $2 \epsilon(1-\epsilon) \in(0,1 / 2)$. McLaughlin, Neuhoff, and Ashley [2] showed that for every $\epsilon \in(0,1 / 2)$, the quadratic

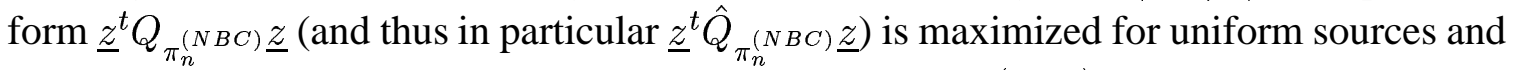
uniform quantizers satisfying $\sum_{i} c_{n}(i)=0$, when $\pi_{n}=\pi_{n}^{(N B C)}$. Shifting the support of a uniform source from $[0,1]$ to $[-1 / 2,1 / 2]$ changes each term in $(7.1)$ by a constant term, independent of the index assignment. Thus $D_{C O}^{\left(\pi_{n}\right)}$ is minimized when $\pi_{n}=\pi_{n}^{(N B C)}$, and therefore the Natural Binary Code is optimal for channel optimized uniform quantizers.

\section{References}

[1] T. R. Crimmins, H. M. Horwitz, C. J. Palermo, and R. V. Palermo, "Minimization of Mean-Square Error for Data Transmitted Via Group Codes," IEEE Transactions on Information Theory, vol. IT-15, pp. 72-78, January 1969.

[2] S. W. McLaughlin, D. L. Neuhoff, and J. J. Ashley, "Optimal Binary Index Assignments for a Class of Equiprobable Scalar and Vector Quantizers," IEEE Transactions on Information Theory, vol. 41, pp. 2031-2037, November 1995.

[3] A. Méhes and K. Zeger, "Binary Lattice Vector Quantization with Linear Block Codes and Affine Index Assignments," IEEE Transactions on Information Theory, vol. 44, no. 1, pp. 79-94, January 1998.

[4] A. Méhes and K. Zeger, "Randomly Chosen Index Assignments Are Asymptotically Bad for Uniform Sources," IEEE Transactions on Information Theory, vol. 45. pp.788-794, March 1999.

[5] R. M. Gray and D. L. Neuhoff, "Quantization," IEEE Transactions on Information Theory, vol. 44. no. 6, pp. 2355-2383, October 1998.

[6] Y. Yamaguchi and T. S. Huang, "Optimum Binary Fixed-Length Block Codes," Quarterly Progress Report 78, M.I.T. Research Lab. of Electronics, Cambridge, Mass., pp. 231-233, July 1965.

[7] T. S. Huang, “Optimum binary code," Quarterly Progress Report 82, M.I.T. Research Lab. of Electronics, Cambridge, Mass., pp. 223-225, July 15, 1966.

[8] H. Kumazawa, M. Kasahara, and T. Namekawa, "A Construction of Vector Quantizers for Noisy Channels," Electronics and Engineering in Japan, vol. 67-B, no. 4, pp. 3947, 1984. 